ciprofloxacin, vancomycin, and metronidazole. The overall death rate was $13 \%$, ranging from $5 \%$ after communityacquired infections to $25 \%$ after infections acquired in the intensive care unit.

It was concluded that most infections treated by surgeons are hospital acquired. Infections with gram-positive cocci and fungi are common, with pulmonary infections becoming more common. Fluoroquinolones have become important therapeutic agents. Depending on the type of practice, these data should be helpful to direct educational efforts so that surgeons can remain knowledgeable and active in the nonsurgical care of their patients.

FROM: Sawyer RG, Raymond DP, Pelletier SJ, Crabtree TD, Gleason TG, Pruett TL. Implications of 2,457 consecutive surgical infections entering year 2000. Ann Surg 2001;233:867-874.

\section{Respiratory Viral Infections in Hospitalized Children: Implications for Infection Control}

Identification of children with respiratory viral infections may augment infection control practices on inpatient units. There are clinical syndromes leading to morbidity among hospitalized children, however, in which a viral etiology of the illness might not be considered. Lichenstein and co-investigators from the Department of Pediatrics, University of Maryland Medical Systems, Baltimore, evaluated virus infection rates among 243 children younger than 1 year to 19 years old hospitalized between October 1993 and April 1994 with asthma, pneumonia, bronchiolitis, fever, apnea, croup, or respiratory distress. Anonymous data collected included admission diagnoses, age, and virus-identification result.

Seventy-one children (29\%) had a virus identified, including $19(15 \%)$ of 123 with asthma, $4(33 \%)$ of 12 with pneumonia, 27 (57\%) of 47 with bronchiolitis, 13 (32\%) of 41 with fever, 4 (44\%) of 9 with apnea, $2(67 \%)$ of 3 with croup, and $2(25 \%)$ of 8 with unspecified respiratory distress.

The authors concluded that this study reinforces the concept that clinicians should consider respiratory viruses for a broad range of diagnoses. This heightened awareness may help reduce the number of nosocomial respiratory viral infections

FROM: Lichenstein R, King JC Jr, Lovchik J, Keane V. Respiratory viral infections in hospitalized children: implications for infection control. South Med J 2002;95:1022-1025.

\section{Risk of Tuberculin Skin Test Conversion Among Healthcare Workers}

Larsen and co-investigators from the Emory University School of Medicine, Atlanta, conducted a prospective observational cohort study to assess rates of and risk factors for tuberculin skin test (TST) conversion among healthcare workers at an urban hospital located in a high tuberculosis-incidence area in 1994-1998. All hospital employees undergoing required testing every 6 months were included.

A total of $69(1.2 \%)$ of 5,773 susceptible employees had a documented TST conversion (overall rate, 0.38 per 100 person-years worked). No significant difference existed in conversion rates among employees with frequent, limited, or no patient contact. Healthcare workers with a TST conversion lived in zip codes with higher tuberculosis case rates $(P \leqslant .05)$. In the multivariate analysis, TST conversion was associated with a history of bacille Calmette-Guerin vaccination (relative risk, 11.63), an annual salary of less than $\$ 20,000$ (relative risk, 3.67), and older age.

In the setting of an effective tuberculosis infection control program, TST conversion rates were low, and risk of conversion among healthcare workers was associated most strongly with nonoccupational factors.

FROM: Larsen NM, Biddle CL, Sotir MJ, White N, Parrott P, Blumberg HM. Risk of tuberculin skin test conversion among health care workers: occupational versus community exposure and infection. Clin Infect Dis 2002;35:796-801.

\section{Mycobacterium simiae Pseudo-Outbreak Resulting From a Contaminated Hospital Water Supply}

El Sahly and colleagues from Baylor College of Medicine, Houston, report that although various species of nontuberculous mycobacteria are known to cause nosocomial pseudo-outbreaks, there have been no detailed reports of nosocomial Mycobacterium simiae pseudo-outbreaks. From April 1997 through February 2001, they recovered $65 M$. simiae isolates from 62 patients at a community teaching hospital in Houston, Texas. The organism was grown in various water samples obtained in the hospital building and in professional building 1 but not in professional building 2, which has a separate water supply system. Thirty-one environmental and human outbreakrelated $M$. simiae isolates had indistinguishable or closely related patterns on pulsed-field gel electrophoresis and were considered clonal.

M. simiae can be a cause of nosocomial pseudo-outbreaks. The reservoir for this pseudo-outbreak was identified as a contaminated hospital water supply.

FROM: El Sahly HM, Septimus E, Soini $\mathrm{H}$, et al. Mycobacterium simiae pseudo-outbreak resulting from a contaminated hospital water supply in Houston, Texas. Clin Infect Dis 2002;35:802-807. 